\title{
Effects of estradiol hormone in menopausal women on anterior disc displacement of temporomandibular joint
}

\author{
Yosaphat Bayu Rosanto* and Poerwati Soetji Rahajoe \\ Departement of Oral and Maxillifacial Surgery, Faculty of Dentistry, Universitas Gadjah Mada, Jl. Denta 1, Sekip Utara, \\ Yogyakarta 55281 Indonesia
}

\begin{abstract}
Anterior disc displacement is a condition when the articular disc is in the anterior normal position. Epidemiological research shows that the prevalence of TMD is higher in women than in men. Women have a dominant hormone that is not owned by men, the hormone estradiol. Estrogen receptors and the hormone progesterone are found in articular joints in humans. Joint structure, extracellular matrix remodeling, and bone volume modification can be affected by sexual hormones. It was thought to cause changes in connective tissue in the TMJ structure. This study aims to determine the effect of the level of the hormone estradiol in menopausal women on anterior temporal disc displacement of the temporomandibular joint. Subjects were 40 postmenopausal women who were examined for temporomandary joints. The diagnosis of anterior disc displacement was made using the Research Diagnostic Criteria for Temporomandibular Disorders (RDC / TMD) method. Subjects were grouped into subjects with normal tempromandibular joints and subjects with anterior disc displacement. Estradiol hormone was examined by the immunoassay method. This study's conclusion is estradiol hormone in menopausal women with anterior disc displacement is higher than menopausal women without anterior disc displacement.
\end{abstract}

Keywords: Estrogen hormone, sexual hormones, temporomandibular disorders, TMD prevalence

\section{Introduction}

Anterior disc displacement is an intracapsular dysfunction that causes disc and articular surfaces degeneration [1]. The first sign of disc displacement is that the patient complains of joint noise or clicking. This condition is caused by stretching of the posterior attachment and the disc being in the anterior condyle at the mouth opening $[2,3,4]$.

Research shows the prevalence of TMD in women 2-5 times higher than in men. Women have a special hormone that is not owned by the pira, the hormone estradiol. The hormone estradiol is one of the most important types of estrogen $[5,6]$.

The mechanism of influence of sexual hormone levels on the emergence of ADD is unknown. Sexual hormones may influence joint structure through changes in the extracellular matrix and bone volume. This is the cause of osteoarthritis in other joints. The study found that the hormones estrogen and progesterone are found in the articular disc. This hormone can increase type III collagen which may cause changes in connective tissue in the joints $[7,8]$. This study aims to determine the effect of the level of the hormone estradiol in menopausal women on anterior temporal disc displacement of the temporomandibular joint.

\section{Materials and methods}

This research is a case control study. Subjects were 40 postmenopausal women who were examined for temporomandary joints. Criteria for inclusion of subjects was there were no vertical dimensions changes marked by occlusion of posterior teeth (premolars and/or molars) on each side, no oral bad habits, no history of trauma to the maxilla and mandible regions, no history of osteoarthritis, do not take steroid or nonsteroidal drugs, and do not use dentures.

The diagnosis of anterior disc displacement was made using the Research Diagnostic Criteria for Temporomandibular Disorders (RDC / TMD) method.

Subjects were grouped into subjects with normal tempromandibular joints (20 subjects) and subjects with anterior disc displacement (20 subjects). All subjects from each group were taken $5 \mathrm{~mL}$ of blood from the radial artery and were examined for the hormone estradiol level by immunoassay in the clinical pathology laboratory. Anterior disc displacement data and estradiol hormone levels were analyzed by paired t-test.

\footnotetext{
* Corresponding author: yosaphatbr@ugm.ac.id
} 


\section{Results}

This study showed the average estradiol levels in subjects with anterior disc displacement was 50.54, with a standard deviation of 33.93. The average estradiol levels in subjects without anterior disc displacement was 32.14 with standard deviation of 22.51 (Figure 1). This showed that the level of estradiol in subjects with anterior disc displacement was higher than the value of estradiol in subjects without anterior disc displacement. Statistical analysis test showed a significance value $P>0.05$. This showed the difference in mean estradiol values in subjects with and without anterior disc displacement was not significant.

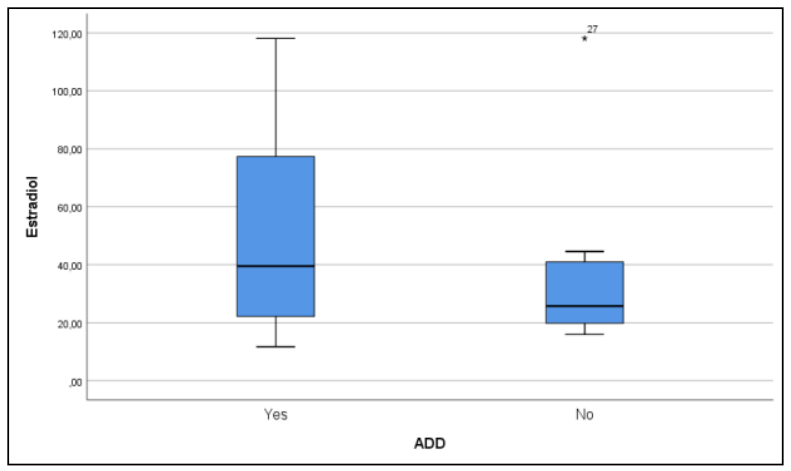

Fig. 1. Graphic of average and deviation standard (SD) of estradiol levels in subjects with anterior disc displacement (ADD) and without ADD. Average estradiol levels in subjects with ADD (Yes) is higher than subjects without ADD (No).

\section{Discussions}

Temporomandibular disorders are clinical conditions that involve masticatory muscles, TMJ, or both [9]. Epidemiological studies show that the prevalence of temporomandibular disorders are higher in women than in men $[5,10,11]$. Women expserience the incidence of TMD 2-5 times higher than men [6]. The purpose of this study is to evaluate the value of the hormone estradiol on anterior disc displacement events. The number of subjects used in this study was 40 , with the criteria of 20 subjects with anterior disc displacement and 20 subjects without anterior disc displacement. The diagnosis of anterior disc displacement was confirmed by the Research Diagnostic Criteria for Temporomandibular Disorders (RDC / TMD) method.

Women have a special hormone that is not owned by the pira, the estradiol hormone. The estradiol hormone is one of the most important types of estrogen. The mechanism of the estradiol hormone affects the incidence of anterior disc displacement can not be known certainly. Joint structure may be influenced by sexual hormones through changes in the extracellular matrix and bone volume. This may be the cause of osteoarthritis in other joints. The study found that the hormones estrogen and progesterone are found in the articular disc. This hormone can increase type III collagen which may cause changes in connective tissue in the joints $[5,6]$.
Joint structure, extracellular matrix remodeling, and bone volume modification can be affected by sexual hormones. These three things are things that happen to anterior disc displacement. Animal studies show that the hormone estrogen modifies inflammation in temporomandibular joint [7]. Estrogen receptors and the progesterone hormone are found in articular joints in humans. This hormone can increase the content of type III collagen which can cause a decrease in the ratio of type I and type III collagen. This is thought to cause connective tissue changes in the TMJ structure $[8,12]$.

Anterior disc displacement in the TMJ is a malrelation disk to the condylar and articular head which can be divided into two categories: anterior disc displacement with reduction and anterior disc displacement without reduction. The condition where the disc is anteriorly and slips back to its normal position during the opening of the mouth is called reduction, the opposite condition is that the displacement of the anterior disk without returning to its normal position is called without reduction. The characteristic of anterior displacement with reduction is clicking when opening and / or closing the mouth $[9,10]$.

This study supports and explains that the incidence of anterior disc displacement occurs more in women than in men. Clicking on the TMJ occurs when there are problems with changes in the relationship of joint ligaments and joint discs. Weak joints in pregnant women can be associated with increased of relaxin hormone [13]. The estradiol hormone in women can also affect the condition of bones, cartilages, and other structures associated with TMJ that can cause anterior disc displacement $[14,15]$. The estradiol hormone can also provide a stimulus to the specific inflammatory response in the joint [16]. The estradiol hormone increases histamine and serotonin in mastocyte cells in periodontal tissue and decreases tamoxifen which is an antagonist of the hormone estradiol.

The results of this study are important to support the clinical examination of anterior disc displacement in women. The estradiol hormone can also aggravate temporomandibular joint damage in studies in mice [17]. Increase in the value of the hormone estradiol in the blood is also associated with an increase in the prevalence of joint pain. Meanwhile, research conducted by Min et al. (2007) stated the opposite results, estradiol hormone deficiency is the cause of TMD events [18]. Research conducted by Yu et al. (2009) stated that the value of the hormone estradiol was not enough to explain TMD predilection in higher women [19]. These studies have shown that there is still much controversy regarding the effect of the hormone estradiol on anterior disc displacement. Other sex hormones can also affect the pathophysiology of TMD and interfere with estrogen. The influence of estrogen on TMD has several aspects that can affect TMD differently [20].

Research conducted in this report shows that menopausal women with anterior disc displacement have higher estradial hormone values (50.54 with a standard deviation of 33.93). Whereas menopausal women without anterior disc displacement had lower estradiol hormone values (32.14 with a standard deviation of 22.51). However, statistically these results 
did not differ statistically. This can be caused by a limited number of subjects. In addition, menopausal women have low estradiol hormone values compared to women who have not experienced menopause yet. This results a non-significant difference, even though the value of the hormone estradiol in the group with anterior disc displacement is higher. In addition, anterior disc displacement is a temporomandibular disorder whose causes are multifactorial. The hormone estradiol is one of the factors that can be the etiology of anterior disc displacement, but anterior disc displacement can also be caused by other things such as trauma and bad habits that affect the joints.

\section{Conclusion}

This research conclude that the average value of the hormone estradiol in menopausal women who experience anterior disc discplacement is higher than the average value of the hormone estradiol in menopausal women who do not experience anterior disc displacement. Various studies have shown different results on the effect of the hormone estradiol to the anterior disc displacement. This study shows that there is an influence of the hormone estradiol on the incidence of anterior disc displacement although it is not so statistically significant. Subsequent studies of the effect of hormones on TMD it is important to use standard TMD examination methods such as magnetic resonance imaging (MRI) to allow comparison of results between studies (eg RDC/TMD).

\section{References}

1. N. Taskaya-Yilmas, M. Ogutcen-Toller, J. Oral Maxillofac. Surg., 59, 860-866 (2001).

2. L. Ji-Young, K. Young-Kyun, K. Su-Gwan, Y. PilYoung, J. Korean Assoc. Oral Maxillofac. Surg., 39, 231-237 (2013).

3. C. Styles, A. Whyte, Br. J. Oral Maxillofac. Surg., 40, 220-228 (2002).

4. K.J. Koh, H.N. Park, K.A. Kim, Imaing. Sci. Dent., 43, 245-251 (2013).

5. D.A. Gancalves, A.L. DalFabbro, J.A. Campos, M.E. Bigal, J.G Speciali, J. Orofac. Pain., 24, 270280 (2010).

6. D. Manfredini, M.B. Bucci, F. Montagna, L. Guarda-Nardini, J. Oral Rehabil., 38, 101-119 (2011).

7. J. Puri, B. Hutchins, L.L. Bellinger, P.R. Kramer, Reprod. Biol. Endocrinol., 7, 155 (2009).

8. D.E. Rizk, E.P. Mensah-Brown, S.I. Chandranath, I. Ahmed, M. Shafiullah, M. Patel, Urol. Res., 31, 147-151 (2003).

9. V. Singh, K.N.V. Sudhakar, S.K. Mallela, R. Mohanty, J. Korean Assoc. Oral Maxillofac. Surg., 43, 368-372 (2017).

10. L.J. Pereira, T. Pereira-cenci, S.M. Pereira, A.A. Cury, G.M. Ambrosano, A.C. Pereira, Braz. Oral Res., 23, 155-160 (2009).
11. M.N. Janal, K.G. Raphael, S. Nayak, J. Klausner, J. Oral Rehabil., 35, 801-809 (2008).

12. A.S. Madani, A.A. Shamsian, M.R. HedayatiMoghaddam, F. Fathi-Moghaddam, M.R. Sabooni, A. Mirmotazavi, M.A. Golmohamadi, J. Oral Rehab., 40, 569-573 (2013).

13. P.C. Conti, J.E. Miranda, A.C. Conti, L.F. Pegoararo, R. Araujo Cdos, J. Appl. Oral Sci., 13, 345-350 (2005).

14. X.X. Kou, Y.W. Wu, Y. Ding, T. Hao, R.Y. Bi, Y.H. Gan, Arthritis Rheum., 63, 1888-1891 (2011).

15. J. Wang, Y. Chao, Q. Wan, Z. Zhu, Med. Hypotheses, 71, 564-567 (2008).

16. B.E. Cairns, J. Oral Rehabil., 37, 391-410 (2010).

17. N.M. Flake, T.O. Hermanstyne, M.S. Gold, Am. J. Physiol. Regul. Integr. Comp. Physiol., 291, 343348 (2006).

18. H.J. Min, M.J. Lee, J.Y. Kim, S.W. Cho, H.D. Park, S.I. Lee, Oral Dis., 13, 220-227 (2007).

19. S. Yu, X. Xing, S. Liang, Z. Ma, F. Li, M. Wang, Med. Hypotheses, 72, 720-722 (2009).

20. M. Berger, L. Szalewski, M. Bakalczuk, G. Bakalczuk, S. Bakalczuk, J. Szkutnik. Prz Menopauzalny, 14, 4, 260-270 (2015). 